\title{
Fluvoxamine for the Early Treatment of SARS-CoV-2 Infection: A Review of Current Evidence
}

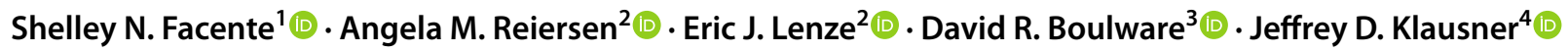

Accepted: 17 October 2021 / Published online: 1 December 2021

(c) The Author(s) 2021

\begin{abstract}
SARS-CoV-2 infection causes COVID-19, which frequently leads to clinical deterioration and/or long-lasting morbidity. Academic and governmental experts throughout the USA met in 2021 to discuss the potential for use of fluvoxamine as early treatment of SARS-CoV-2 infection. Fluvoxamine is a selective serotonin reuptake inhibitor (SSRI) that is a strong sigma-1 receptor agonist, and this may effectively reduce cytokine production, preventing clinical deterioration. This repurposed psychiatric medication has a well-known safety record, is inexpensive, easy to use, and widely available, all of which are advantages during this global COVID-19 pandemic. At the meeting, experts reviewed the existing published literature on the use of fluvoxamine as experimental COVID-19 treatment, as well as prior research on the potential mechanisms for anti-inflammatory effects of fluvoxamine, including for other conditions including sepsis. Investigators shared current trials underway and existing gaps in knowledge. Two randomized controlled trials and one observational study examining the effect of fluvoxamine in COVID-19 treatment have found high efficacy. Four larger randomized clinical trials are currently underway, including three in the USA and Canada. More data are needed on dosing and mechanisms of effect; however, fluvoxamine appears to have substantial potential as a safe and widely available medication that could be repurposed to ameliorate serious COVID-19-related morbidity and mortality. As of April 2021, fluvoxamine was mentioned in the NIH COVID-19 treatment guidelines, although no recommendation is made for or against use. Available data may warrant clinician discussion of fluvoxamine as a treatment option for COVID-19, using shared decision making.
\end{abstract}

Shelley N. Facente

sfacente@berkeley.edu

$\triangle$ Jeffrey D. Klausner

jdklausner@med.usc.edu

1 University of California, 2121 Berkeley Way West, 5th floor, Berkeley, CA 94720, USA

2 Washington University in St. Louis, St. Louis, MO, USA

3 University of Minnesota, Minneapolis, MN, USA

4 University of Southern California, Los Angeles, CA, USA

\section{Key Points}

Fluvoxamine appears to have potential as a safe, inexpensive, and widely available medication that could be effectively repurposed to ameliorate serious COVID19-related morbidity and mortality.

Fluvoxamine prevented clinical deterioration and longlasting symptoms related to COVID-19 in initial studies and one large clinical trial, with several large clinical trials underway globally.

Current information may warrant clinician discussion of fluvoxamine as a treatment option for COVID-19, using shared decision making. 


\section{Introduction}

SARS-CoV-2 infection causes coronavirus disease 2019 (COVID-19), which frequently leads to clinical deterioration around the second week of illness [1, 2], and/or longlasting morbidity after initial infection [3, 4]. While some treatment options have come to light for those who have already progressed to severe disease, increasingly there are calls for discovery of effective early treatments that can prevent clinical deterioration and/or long-term morbidity in the first place [5].

Evidence is steadily accumulating that points to the role of excessive immune response in SARS-CoV-2 infection as a key factor in clinical deterioration or long-term symptoms. A leading theory is that SARS-CoV-2 replicates in an intermediate compartment between the endoplasmic reticulum (ER) and Golgi complex, leading to ER stress and increased cytokine production causing an excessive inflammatory response [6]. In late 2020, Gordon and colleagues [7] identified that knockout or knockdown of SIGMARl gene, which encodes the sigma-1 receptor (S1R), caused substantial reduction in SARS-CoV-2 replication. The S1R is already well established as influencing the pathophysiology of multiple psychiatric, neurodegenerative, and central nervous system disorders [8-12]. The findings of Gordon and colleagues, along with prior research indicating a potential role for S1R ligands in preventing sepsis-also associated with excessive inflammatory cytokine production [13] -has led to an interest in exploring the potential for the repurposing of existing drugs that target the S1R as early treatment for SARS-CoV-2 infection.

Fluvoxamine is a selective serotonin reuptake inhibitor (SSRI) that has been widely used globally since the 1990s. Previous studies have shown high affinity for the S1R [14], and in multiple comparative studies fluvoxamine has been consistently ranked as one of the most potent S1R agonists clinically available $[6,10,15]$; therefore, it may effectively reduce cytokine production and prevent clinical deterioration. It is inexpensive, easy to use, widely available globally, and highly lipophilic, with rapid intracellular uptake into lung epithelial cells [16].

\subsection{Safety Profile}

Fluvoxamine has a well-known safety profile $[17,18]$, with nausea as the most common adverse event. In a global database of 35,368 people who had taken fluvoxamine across 66 studies in 11 countries, nausea was reported in $15.7 \%$ of patients, followed by somnolence $(6.4 \%)$, asthenia $(5.1 \%)$, headache $(4.8 \%)$, and dry mouth $(4.8 \%)$. Serious adverse events occurred in approximately $2.0 \%$ of people who were taking this medication for a psychiatric disorder, with $1.6 \%$ requiring hospitalization and $<0.4 \%$ experiencing another serious adverse event, including a suicide attempt, depression, death, or accidental injury [17].

\section{Current Evidence on Fluvoxamine as COVID-19 Treatment}

Several studies have examined the efficacy of fluvoxamine for improvement in the clinical progression of SARSCoV-2 infection or have explored the potential mechanisms by which fluvoxamine may have a beneficial effect as early COVID-19 treatment (Table 1). In a small randomized clinical trial known as STOP COVID, participants were randomly assigned to receive $100 \mathrm{mg}$ of fluvoxamine $(n=80)$ or placebo $(n=72)$ up to three times per day, for 15 days [1]. Lenze and colleagues hypothesized that fluvoxamine would prevent clinical deterioration if prescribed early in COVID-19 (i.e., within 7 days of symptoms onset). All participants were aged $\geq 18$ years, were not already hospitalized or living in an institutional setting (but were self-isolated per public health guidance), and had diagnosed, symptomatic SARS-CoV-2 infection, with symptoms onset of a median of 4 days prior to enrollment (interquartile range, 3-5; maximum 7 days). The primary endpoint of this study was clinical deterioration, as defined by both (1) presence of dyspnea (i.e., shortness of breath) or hospitalization for dyspnea or pneumonia, and (2) presence of hypoxia with pulse oxygen saturation $<92 \%$ on room air. At study completion, none $(0 \%)$ of the 80 participants receiving fluvoxamine met the criteria for clinical deterioration, but $6(8.3 \%)$ of the 72 participants receiving placebo deteriorated clinically (absolute difference from survival analysis $8.7 \%, 95 \% \mathrm{CI}$, $1.8 \%$ to $16.4 \%$; log-rank $p=0.009$ ). Despite the high dose of fluvoxamine administered during this trial, only one serious adverse event occurred, of a person with no respiratory deterioration, hospitalized for dehydration. Overall, 11 other adverse events occurred in the treatment group; the placebo group had 6 serious adverse events (all hospitalizations for COVID-19) and 12 other adverse events, including higher reports of pneumonia and gastrointestinal symptoms.

Inspired by the newly published results from Lenze and colleagues, during a large occupational outbreak of COVID19 in late 2020, Seftel and Boulware conducted a real-world observational study of early SARS-CoV-2 treatment with fluvoxamine [19]. A total of 113 people in a work-associated congregate living environment were offered fluvoxamine on the same day that they tested positive for SARS-CoV-2. Testing used a rapid antigen test during one of three rounds of mass testing during the outbreak, regardless of symptoms. Participants could choose whether or not to accept the medication, given the limited data in support of its use for this purpose. A total of 65 people (57.5\%) opted for treatment 


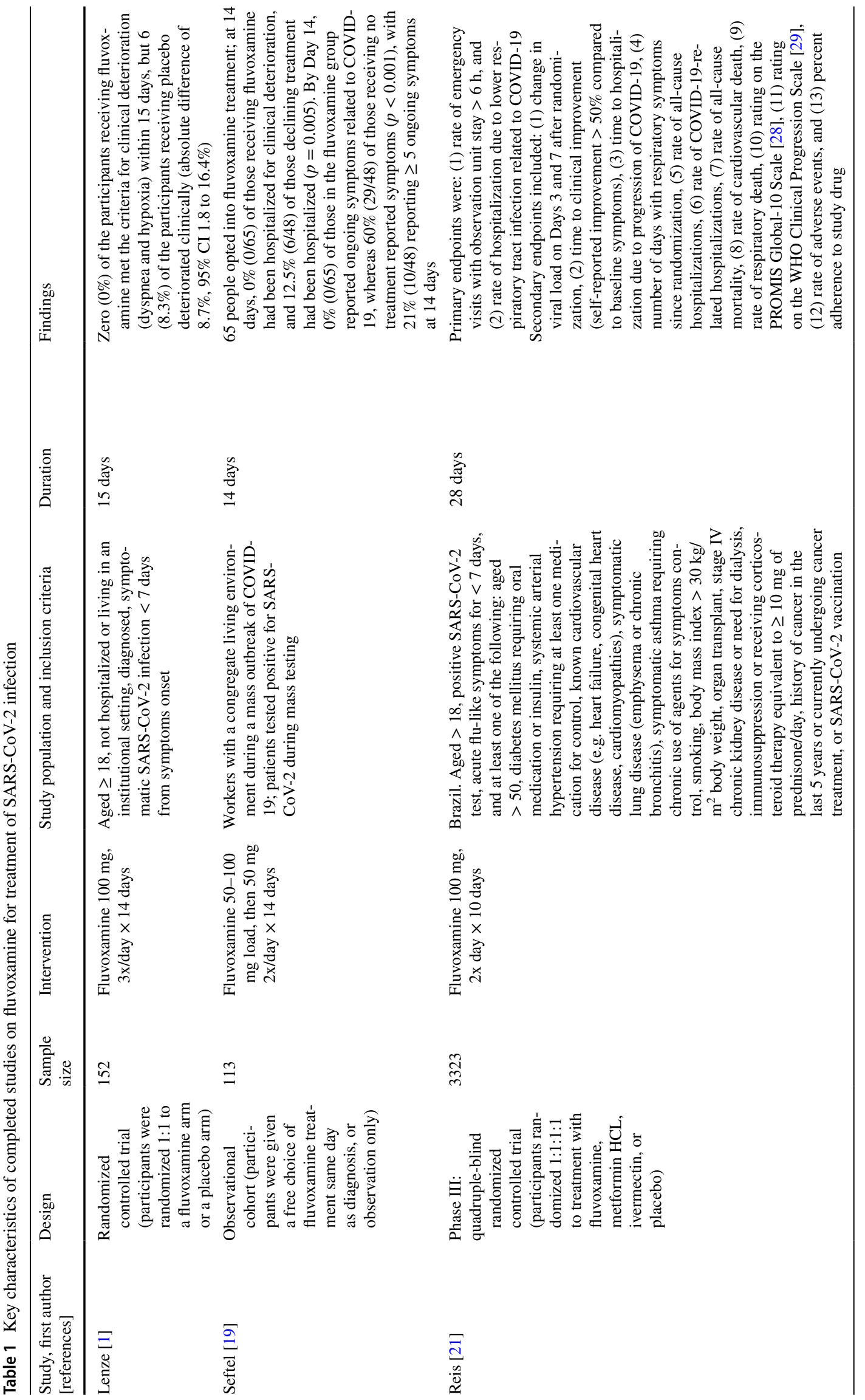


with a $50-100 \mathrm{mg}$ loading dose of fluvoxamine, followed by $50 \mathrm{mg}$ twice daily $(50 \mathrm{mg}$ was a lower dose than the Lenze trial), and 48 (42.5\%) opted for observation alone. Participants received in-person follow-up at 7 and 14 days, with $100 \%$ retention. At 14 days, no patients receiving fluvoxamine had been hospitalized for clinical deterioration; however, $12.5 \%$ (6/48) of patients who declined treatment had been hospitalized ( $p=0.005$ ). Notably, by Day $14,0 \%$ $(0 / 65)$ of those in the fluvoxamine group reported ongoing symptoms related to SARS-CoV-2 infection, whereas $60 \%$ (29/48) of those receiving no treatment reported symptoms $(p<0.001)$, with $21 \%(10 / 48)$ reporting five or more ongoing symptoms at 14 days.

Most recently, investigators from the TOGETHER trial in Brazil published results of their Phase III clinical trial. The trial comprised four treatment arms aimed to evaluate the effect of various medications to reduce the need for emergency care requiring observation for $>6 \mathrm{~h}$ due to the worsening COVID-19, and/or hospitalization due to COVID-19-related lower respiratory tract infection. Adults with a positive SARS-CoV-2 test, acute flu-like symptoms for $<7$ days, and at least one enhancement factor (e.g., older age, diabetes mellitus, immunosuppression, etc.) were randomized to 10 days of treatment in one of four treatment arms: fluvoxamine (100 mg twice daily), metformin, ivermectin, or placebo. At the second interim analysis in April 2021, metformin was dropped, yet fluvoxamine was retained [20], and on August 6, 2021, the trial arms were stopped for superiority of fluvoxamine, with a total of 3323 patients enrolled. The relative risk (RR) of hospital admittance or emergency room observation for more than 6 hours was determined to be 0.68 (95\% Bayesian Credible Interval [BCI] 0.52-0.88) for participants receiving fluvoxamine versus the placebo control in the intention-to-treat sample. In a per-protocol analysis of participants who were adherent to at least $80 \%$ of pills, fluvoxamine was effective against both deterioration and mortality, with an RR of 0.34 for hospitalization (95\% BCI 0.21-0.54) and an odds ratio of 0.09 (95\% CI 0.01-0.47) for mortality [21].

Further study is needed to investigate potential anti-viral and anti-inflammatory, and other mechanisms of fluvoxamine in the context of SARS-CoV-2 infection. One theory of its mechanism of action is a reduction in the ER stress response and reduction in cytokine production as a result of sigma-1 activation, given fluvoxamine's potency as a S1R agonist. The $\mathrm{S} 1 \mathrm{R}$ is an ER chaperone protein that regulates the ER stress response as well as production of cytokines in response to infection and other inflammatory triggers; S1R agonists prevent Inositol Requiring Enzyme $1 \alpha$ (IRE1) from activating X-Box Binding Protein-1 (XBP-1) mRNA, therefore regulating the ER stress response, and reducing cytokine production [13]. In a recent study of affinity of various antidepressant drugs for the S1R in rat brains, Ishima and colleagues found that fluvoxamine was the most potent S1R agonist among ten different antidepressants tested [14]. In a 2019 study of fluvoxamine's effectiveness in preventing lethal septic shock in mice, Rosen and colleagues found that only $9 \%$ of wild-type (WT) mice died after injection with lipopolysaccharide (LPS), known to rapidly induce proinflammatory cytokines in mice and humans, compared to $62 \%$ of mice with sigma- 1 knockout (KO). Similar results were found after infection with fecal slurry $(1 \mathrm{~g} / \mathrm{kg}$ of bodyweight), which resulted in septic shock and death in substantially more of the S1R KO mice than WT mice $(p<0.05)$. However, in the presence of an IRE1 inhibitor, survival rates of S1R KO mice and WT mice were similar, further demonstrating the potential mechanistic effects of S1R agonism to dampen inflammatory response [13].

Rather than just anti-inflammatory effects, another possible mechanism of fluvoxamine's effect is antiviral properties through lysosomotropic effects [22]. Cationic amphiphilic drugs (CADs) such as fluvoxamine tend to accumulate in the lysosomes, altering the $\mathrm{pH}$, interfering with viral proteins that accumulate, and/or preventing mature virus from using lysosomes to escape the cell, which is one characteristic of coronaviruses [23]. It is also possible that lysosomotropic antidepressants may interfere with viral entry through inhibition of acid sphingomyelinase activity [24]. Functional inhibitors of acid sphingomyelinase (FIASMAs) prevent sphingomyelin from being converted to ceramide, which makes it more difficult for a virus like SARS-CoV-2 to enter the cell [25].

Others have theorized that the inhibition of platelet aggregation and mast cell degranulation may be likely mechanisms of action [26, 27]. SSRIs like fluvoxamine have been shown to decrease platelet aggregation and increase bleeding time. Since hyperactive platelets can release excessive serotonin, and serotonin clearance requires healthy pulmonary endothelium, beginning SSRIs early in SARS-CoV-2 infection-before the pulmonary endothelium is damaged by COVID-19-may prevent the effect of potentially damaging platelet hyperactivation, inflammatory thrombosis, and platelet serotonin storm, therefore reducing the risk of a hyperserotonergic state leading to acute respiratory distress $[26,27]$. Furthermore, SSRIs decrease histamine release from human mast cells, and reduce mRNA levels of protease-1 in mast cells; this is important, as postmortem lung biopsies of patients with COVID-19 have linked activated mast cells to pulmonary thromboses and edema [26].

\section{Additional Studies Underway}

On December 17, 2020, Lenze and colleagues began a new nationwide, fully remote (internet-based) Phase III randomized controlled trial named STOP COVID 2 
(StopCovidTrial.com; ClinicalTrials.gov: NCT04668950) to confirm the initial results from their preliminary trial. This trial is taking place in the USA and Canada, with nation-wide internet-based enrollment and telemedicine appointments for all study interactions. The preliminary trial (STOP COVID) resulted in few cases of clinical deterioration overall due to limited sample size and relatively young and healthy participants, resulting in low precision of the effect size estimate [1]. STOP COVID 2 aimed to recruit 1100 participants with eligibility criteria similar to the first trial (SARS-CoV-2 positive and symptomatic within 7 days of symptoms onset, residing in the community rather than in a hospital or other institutional setting); however, in this trial the sample was enriched, with participants needing to have one or more of the following risk factors for more severe COVID-19: aged $\geq 40$ years, obesity, diabetes, hypertension, heart disease, lung disease, an immune condition, and/ or being African American, Latinx, or Native American.

On May 19, 2021, STOP COVID 2 stopped enrolling new participants on the advice of the Data and Safety Monitoring Board, based on an overall lower rate of clinical deterioration than anticipated (leading to a much larger sample size necessary to observe the a priori minimum detectable effect), the decreasing number of volunteers enrolling in the trial, and their review of the unblinded interim results to date. There were no adverse safety signals, but due to successful vaccination roll-out in the USA and Canada, the trial was no longer expected to accrue the needed number of participants. Participants already enrolled in the trial finished their assigned doses of therapy and will complete the planned follow-up questionnaires at 15 and $~ 90$ days to assess short- and long-term secondary outcomes. This trial has the same duration and primary outcome as the initial trial (clinical deterioration within 15 days of enrollment, defined by dyspnea and hypoxia), but has lower dosing: 100 $\mathrm{mg}$ twice/day, instead of three times daily for 15 days, of either fluvoxamine or placebo. With enrollment halted, the trial will not have the required sample size and statistical power to detect an effect of fluvoxamine on the primary outcome. However, this trial also includes a secondary outcome of health functioning and symptoms assessed at 15 days and again at 3 months, measured by the Global Health Scale [28], and an exploratory symptom questionnaire to assess any effect of fluvoxamine on long-term COVID-19 morbidity.

In January 2021, Drs Bramante, Boulware, and colleagues at the University of Minnesota; Northwestern University; University of Colorado, Denver; UCLA Olive View; and OptumLabs began stage 1 of a Phase III, factorial randomized clinical trial known as COVID-19-OUT (covidout. com; ClinicalTrials.gov: NCT04510194). The initial stage of the quadruple-blinded trial enrolled 70 patients, with the fully enrolled trial having a planned 1160 participants. This factorial trial has five experimental arms (fluvoxamine only, metformin only, ivermectin only, metformin plus fluvoxamine, or metformin plus ivermectin), with one placebo arm. Adults aged 30 to 85 years are eligible for inclusion within $\leq 3$ days of a positive PCR test for SARS-CoV-2 infection if they are asymptomatic or have had symptoms for $<7$ days before randomization, enroll within 3 days of testing, have a body mass index $\geq 25 \mathrm{~kg} / \mathrm{m}^{2}$ by self-report height/ weight or $\geq 23 \mathrm{~kg} / \mathrm{m}^{2}$ for patients who self-identify as South Asian or Latinx. A glomerular filtration rate (GFR) will be obtained in persons older than age 75 years or who have a history of heart, kidney, or liver failure if a GFR is not visible within the electronic health record within 2 weeks, to ensure these high-risk individuals have a GFR $>45 \mathrm{~mL} / \mathrm{min}$. The primary outcome measures are (1) decreased oxygenation at 14 days (defined as pulse oxygen saturation $\leq 93 \%$ on home monitoring), (2) emergency department utilization for COVID-19 symptoms at 14 days (and/or hospitalization/ death), and (3) post-acute sequelae of SARS-CoV-2 infection (PASC) assessment at 6 and 12 months. This trial is currently enrolling.

Another trail also enrolling is ACTIV-6 (ClinicalTrials. gov: NCT04885530), which is a Phase III, placebo-controlled, randomized trial, run by Dr. Naggie at Duke Clinical Research Institute. This trial has three experimental arms (ivermectin, fluvoxamine, and fluticasone), with a placebo comparator arm matched to each experimental arm. Both participants and the study teams know which study drug they have been allocated but are blinded to whether they are in the experimental or placebo comparator arms for that study drug. Adults aged $\geq 30$ years with SARS-CoV-2 infection confirmed within 10 days of study screening with two or more current symptoms of acute SARS-CoV-2 infection (fatigue, dyspnea, fever, cough, nausea, vomiting, diarrhea, body aches, chills, headache, sore throat, nasal symptoms, or new loss of sense of taste or smell) began enrolling in this study on June 8, 2021, with a goal of enrolling 15,000 participants before a primary completion date of December 2022. The primary outcome measures of this trial are the number of hospitalizations, number of deaths, and number of symptoms within 14 days, as measured by patient reports.

Finally, another randomized controlled trial of fluvoxamine and COVID-19 is underway in Hungary: SigmaDrugs Research Ltd. is currently recruiting for a Phase II trial (ClinicalTrials.gov: NCT04718480), studying the time to clinical recovery after treatment with 74 days of fluvoxamine $100 \mathrm{mg}$ taken twice daily, compared to placebo. Up to 100 adults who have moderately severe cases of COVID-19 (having each of the following: dyspnea without respiratory distress, a respiration rate 22-29 times per minute, resting pulse oxygen saturation $\geq 93 \%$, and pneumonia with pulmonary infiltrates occupying $\leq 50 \%$ of the lung-fields) will be enrolled. The primary endpoint of clinical recovery includes 


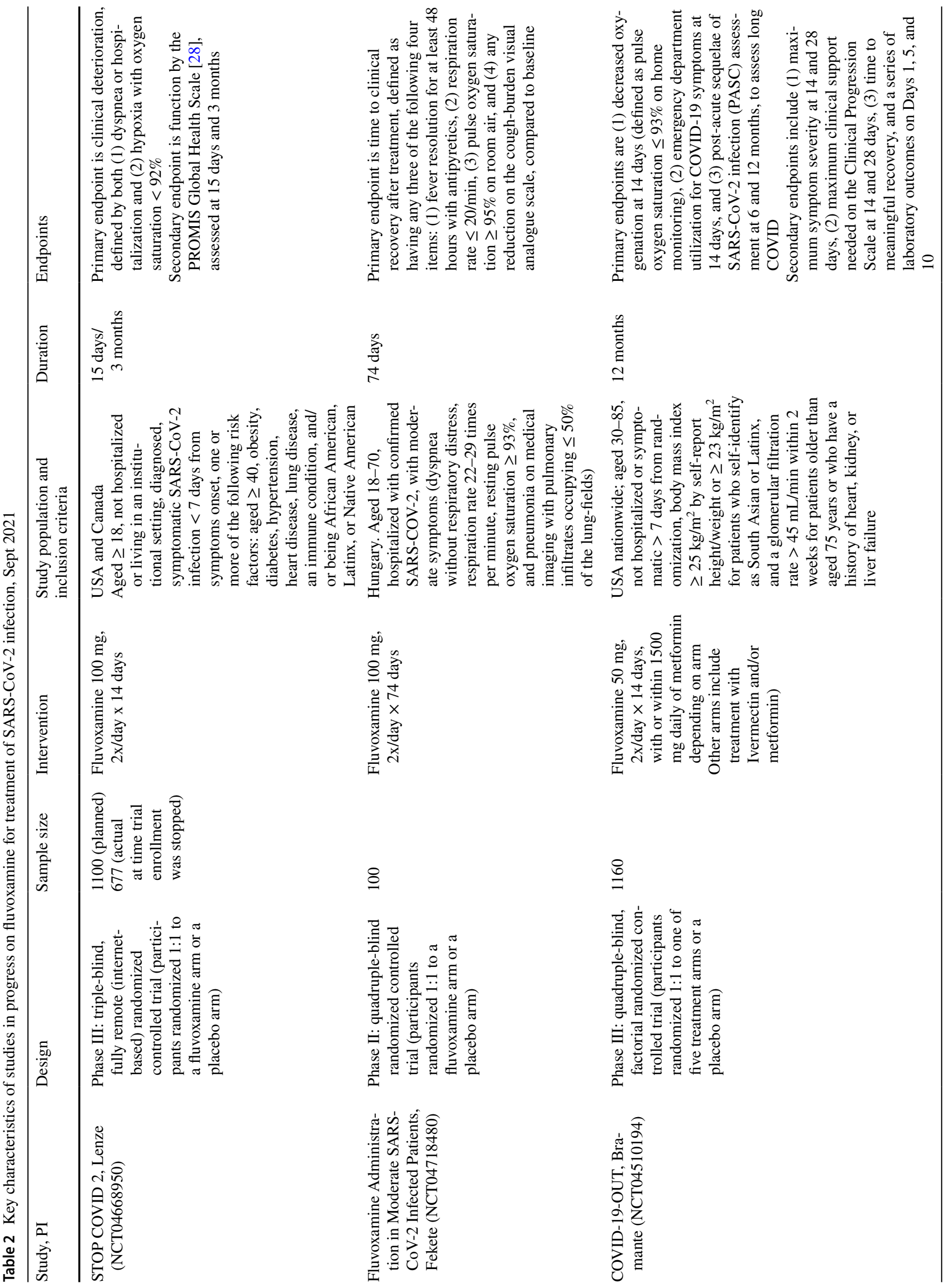




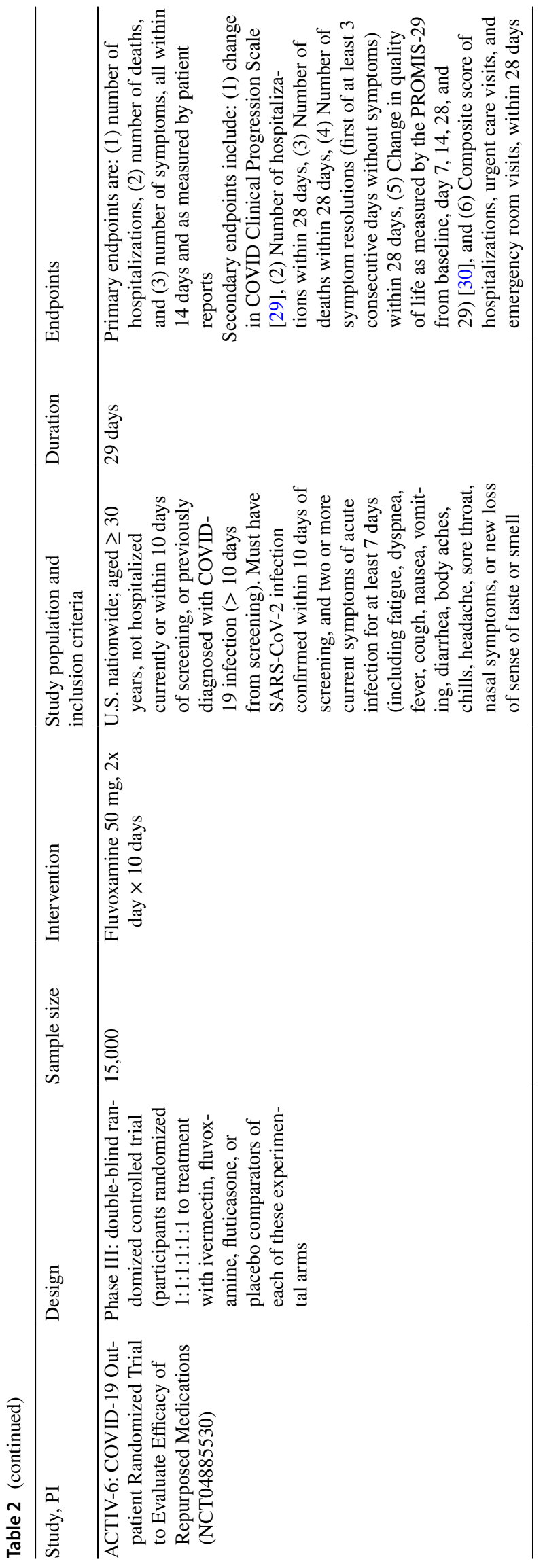

resolving to normal any three of the following four clinical indicators: fever, respiratory rate, pulse oxygen saturation, and cough burden. This study has an estimated completion date of December 2021.

Multiple studies of the mechanisms of fluvoxamine's effect on SARS-CoV-2 are also currently underway, including in vitro and animal studies at multiple institutions. Publication of findings from these pending studies is eagerly awaited, as they will offer meaningful contributions to our understanding of how and why early treatment with fluvoxamine may have a beneficial effect on COVID-19-related morbidity and mortality.

\section{Knowledge Gaps and Challenges}

The studies described above (Table 2) are designed to address two key gaps in knowledge, which must be better understood to inform widespread use of fluvoxamine as early treatment for COVID-19.

\subsection{Efficacy}

With publication of the TOGETHER trial results, along with the first STOP COVID trial, the efficacy of fluvoxamine for preventing clinical deterioration and/or long-term morbidity due to SARS-CoV-2 infection has been demonstrated in two randomized placebo-controlled trials. The extent of fluvoxamine's efficacy remains unclear; in both the STOP COVID and Seftel and Boulware studies, no participant who received fluvoxamine experienced the primary adverse outcome-respiratory deterioration (in the case of STOP COVID) or hospitalization (Seftel and Boulware) - for their COVID-19 infection, compared with $8.3 \%$ or $12.5 \%$ of participants in the control arm, respectively. Similarly, in the TOGETHER trial per-protocol analysis, the fluvoxamine arm was associated with a $66 \%$ reduction in clinical deterioration requiring hospitalization or extended emergency contact, and a $91 \%$ reduction in mortality [21]. In addition to these primary endpoints, the Seftel study also found that zero participants in the fluvoxamine group had ongoing symptoms at 14 days, compared to $60 \%$ of people in the control group; however, STOP COVID found no differences in short-term symptomatic recovery and saw some lingering symptoms reported after 4 months in both the fluvoxamine and placebo arms, although numbers were too small to allow for statistical comparisons. While mortality due to COVID-19 has received the most attention to date among the media and general public, both short-term and long-term morbidity from SARS-CoV-2 infection is a substantial burden worldwide. 
Fig. 1 Recommendations for research activities
- Clinical efficacy: It is imperative that these promising preliminary results are challenged in a larger clinical trial, to test the robustness of these findings. Further studies are needed to collect serial cytokine and virologic data for patients randomized to fluvoxamine prescription compared to placebo, which will also further our understanding of how the drug may be working relative to the clinical outcomes observed in the existing studies. Fluvoxamine should also be tested later in the course of COVID-19, as it may be beneficial in preventing further deterioration in those already hospitalized, as has been shown with dexamethasone.

- Dosing strategies: The optimal dosing of fluvoxamine to maximize effect while minimizing toxicity is still under investigation. Particularly, further exploration is needed regarding the best methods for offering fluvoxamine to patients with SARS-CoV-2 infection if they are already taking a different SSRI. In the STOP COVID trial, people already on SSRIs are not excluded, but risk of serotonin syndrome is assessed on a case-by-case basis. Given the high prevalence of SSRI uptake in the U.S. and around the world, this will be an important consideration if the effects of fluvoxamine on SARSCoV-2 prognosis persist in further studies.

- Mechanisms of action: The findings of the preliminary human studies of fluvoxamine are quite impressive, and the safety and logistical ease of fluvoxamine prescription early in SARS-CoV-2 infection is attractive. However, the mechanism of effect is not well understood, and more data is needed to understand why and how this repurposed medication appears to have such a substantial impact on COVID-19.

\subsection{Mechanisms of Action}

The anti-inflammatory effects of fluvoxamine are well understood, but the mechanism by which it could have such a strong effect on morbidity and mortality-especially without more clearly demonstrated anti-viral effects-is still unclear. The in vitro and animal studies currently underway will greatly improve our understanding of the biological effect of this drug, and further explore any potential for the prolongation of the viral phase of infection as a result of fluvoxamine's inhibition of the inflammatory response, which would warrant caution with its use. Since fluvoxamine seems to have anti-inflammatory and immune modulatory actions without substantial suppression of the immune response, it may be much safer to use in the earliest stages of COVID-19, as compared to systemic steroids.

\section{Research Priorities and Future Directions}

As of April 2021, fluvoxamine is now mentioned in the NIH COVID-19 treatment guidelines, although no recommendation is yet made for or against use, due to insufficient available evidence. Based on our review of the current evidence and studies in progress, there are several key research priorities (Fig. 1). In summary, fluvoxamine is seen as a highly promising drug for COVID-19, but more studies are needed to elucidate its mechanism of action and possible deleterious effects.

\section{Declarations}

Funding This report was supported in part by a gift to the Keck School of Medicine of the University of Southern California by the W.M. Keck Foundation.

Competing interests Dr. Reiersen and Dr. Lenze are listed on a patent application related to methods of treating COVID-19, that was filed by Washington University in St. Louis. No other authors report any conflicts of interest.

Availability of data and material As this is a narrative review of available scientific evidence, all included data and material are available through the cited literature.

Code availability Not applicable.

Author contributions SNF: Overall synthesis of material and first draft of manuscript; AMR, EJL, DRB: conceptualization, curatorship of data and findings; JDK: conceptualization, project leadership, and funding. All authors read and approved the manuscript.

Open Access This article is licensed under a Creative Commons Attribution-NonCommercial 4.0 International License, which permits any non-commercial use, sharing, adaptation, distribution and reproduction in any medium or format, as long as you give appropriate credit to the original author(s) and the source, provide a link to the Creative Commons licence, and indicate if changes were made. The images or other third party material in this article are included in the article's Creative Commons licence, unless indicated otherwise in a credit line to the material. If material is not included in the article's Creative Commons licence and your intended use is not permitted by statutory regulation or exceeds the permitted use, you will need to obtain permission directly from the copyright holder. To view a copy of this licence, visit http://creativecommons.org/licenses/by-nc/4.0/. 


\section{References}

1. Lenze EJ, Mattar C, Zorumski CF, et al. Fluvoxamine vs placebo and clinical deterioration in outpatients with symptomatic COVID-19: a randomized clinical trial. JAMA. 2020;324(22):2292-300. https://doi.org/10.1001/jama.2020. 22760.

2. Cummings MJ, Baldwin MR, Abrams D, et al. Epidemiology, clinical course, and outcomes of critically ill adults with COVID-19 in New York City: a prospective cohort study. Lancet. 2020;395(10239):1763-70. https://doi.org/10.1016/S01406736(20)31189-2.

3. Marshall M. The lasting misery of coronavirus long-haulers. Nature. 2020;585(7825):339-41. https://doi.org/10.1038/ d41586-020-02598-6.

4. Del Rio C, Collins LF, Malani P. Long-term Health Consequences of COVID-19. JAMA. 2020. https://doi.org/10.1001/jama.2020. 19719.

5. Kim PS, Read SW, Fauci AS. Therapy for early COVID-19: a critical need. JAMA. 2020;324(21):2149-50. https://doi.org/10. 1001/jama.2020.22813.

6. Hashimoto K. Repurposing of CNS drugs to treat COVID-19 infection: targeting the sigma-1 receptor. Eur Arch Psychiatry Clin Neurosci. 2021. https://doi.org/10.1007/s00406-020-01231-x.

7. Gordon DE, Hiatt J, Bouhaddou M, et al. Comparative hostcoronavirus protein interaction networks reveal pan-viral disease mechanisms. Science. 2020. https://doi.org/10.1126/science.abe94 03.

8. Hayashi T, Su TP. An update on the development of drugs for neuropsychiatric disorders: focusing on the sigma 1 receptor ligand. Expert Opin Ther Targets. 2008;12(1):45-58. https://doi.org/10. 1517/14728222.12.1.45.

9. Albayrak Y, Hashimoto K. Sigma-1 receptor agonists and their clinical implications in neuropsychiatric disorders. Adv Exp Med Biol. 2017;964:153-61. https://doi.org/10.1007/978-3-31950174-1_11.

10. Hashimoto K. Activation of sigma-1 receptor chaperone in the treatment of neuropsychiatric diseases and its clinical implication. J Pharmacol Sci. 2015;127(1):6-9. https://doi.org/10.1016/j.jphs. 2014.11.010.

11. Hashimoto K. Targeting the sigma-1 receptor chaperone in the treatment of perinatal brain injury. Exp Neurol. 2015;265:118-21. https://doi.org/10.1016/j.expneurol.2014.12.019.

12. Couly S, Goguadze N, Yasui Y, et al. Knocking out sigma-1 receptors reveals diverse health problems. Cell Mol Neurobiol. 2020. https://doi.org/10.1007/s10571-020-00983-3.

13. Rosen DA, Seki SM, Fernández-Castañeda A, et al. Modulation of the sigma-1 receptor-IRE1 pathway is beneficial in preclinical models of inflammation and sepsis. Science Transl Med. 2019. https://doi.org/10.1126/scitranslmed.aau5266.

14. Ishima T, Fujita Y, Hashimoto K. Interaction of new antidepressants with sigma-1 receptor chaperones and their potentiation of neurite outgrowth in PC12 cells. Eur J Pharmacol. 2014;727:16773. https://doi.org/10.1016/j.ejphar.2014.01.064.

15. Cobos EJ, Entrena JM, Nieto FR, Cendán CM, Del Pozo E. Pharmacology and therapeutic potential of sigma(1) receptor ligands. Curr Neuropharmacol. 2008;6(4):344-66. https://doi.org/10.2174/ 157015908787386113
16. Hallifax D, Houston JB. Saturable uptake of lipophilic amine drugs into isolated hepatocytes: mechanisms and consequences for quantitative clearance prediction. Drug Metab Dispos. 2007;35(8):1325-32. https://doi.org/10.1124/dmd.107.015131.

17. Wagner W, Zaborny BA, Gray TE. Fluvoxamine. A review of its safety profile in world-wide studies. Int Clin Psychopharmacol. 1994;9(4):223-7. https://doi.org/10.1097/00004850-19940 0940-00001.

18. Altamura AC, Caldiroli A, Buoli M. Pharmacokinetic evaluation of fluvoxamine for the treatment of anxiety disorders. Expert Opin Drug Metab Toxicol. 2015;11(4):649-60. https://doi.org/10.1517/ 17425255.2015 .1021331$.

19. Seftel D, Boulware DR. Prospective cohort of fluvoxamine for early treatment of COVID-19. Open Forum Infect Dis. 2021. https://doi.org/10.1093/ofid/ofab050.

20. Together Clinical Trials [Website]. http://togethertrial.com. Accessed 3 Jun 2021.

21. Reis G, dos Santos Moreira Silva EA, Medeiros Silva DC, Thabane L, Milagres AC, Santiago Ferreira T, et al. Effect of early treatment with Fluvoxamine on risk of emergency care and hospitalization among patients with COVID-19: The TOGETHER randomized platform clinical trial. Lancet Glob Health 2021;S2214109X(21)00448-4. https://doi.org/10.1016/S2214-109X(21) 00448-4.

22. Homolak J, Kodvanj I. Widely available lysosome targeting agents should be considered as potential therapy for COVID-19. Int J Antimicrob Agents. 2020;56(2): 106044. https://doi.org/10. 1016/j.ijantimicag.2020.106044.

23. Ghosh S, Dellibovi-Ragheb TA, Kerviel A, et al. $\beta$-Coronaviruses use lysosomes for egress instead of the biosynthetic secretory pathway. Cell. 2020;183(6):1520-35.e14. https://doi.org/10. 1016/j.cell.2020.10.039.

24. Carpinteiro A, Edwards MJ, Hoffmann M, et al. Pharmacological inhibition of acid sphingomyelinase prevents uptake of SARSCoV-2 by epithelial cells. Cell Rep Med. 2020;1(8): 100142. https://doi.org/10.1016/j.xcrm.2020.100142.

25. Kornhuber J, Muehlbacher M, Trapp S, et al. Identification of novel functional inhibitors of acid sphingomyelinase. PLoS ONE. 2011;6(8): e23852. https://doi.org/10.1371/journal.pone.0023852.

26. Sukhatme VP, Reiersen AM, Vayttaden SJ, Sukhatme VV. Fluvoxamine: a review of its mechanism of action and its role in COVID-19. Front Pharmacol. 2021;12: 652688. https://doi.org/ 10.3389/fphar.2021.652688.

27. Hashimoto Y, Suzuki T, Hashimoto K. Old drug fluvoxamine, new hope for COVID-19. Eur Arch Psychiatry Clin Neurosci. 2021. https://doi.org/10.1007/s00406-021-01326-z.

28. Hays RD, Bjorner JB, Revicki DA, Spritzer KL, Cella D. Development of physical and mental health summary scores from the patient-reported outcomes measurement information system (PROMIS) global items. Qual Life Res. 2009;18(7):873-80. https://doi.org/10.1007/s11136-009-9496-9.

29. WHO Working Group on the Clinical Characterisation and Management of COVID-19 Infection. A minimum common outcome measure set for COVID-19 clinical research. Lancet Infect Dis. 2020. https://doi.org/10.1016/S1473-3099(20)30483-7.

30. Cella D, Choi SW, Condon DM, Schalet B, Hays RD, Rothrock NE, et al. PROMIS ${ }^{\circledR}$ adult health profiles: efficient short-form measures of seven health domains. Value Health. 2019;22(5):53744. https://doi.org/10.1016/j.jval.2019.02.004. 